\title{
Strategies for the analysis of osteitic bone defects at the diaphysis of long bones
}

\author{
A. H. Tiemann · H. G. K. Schmidt • \\ R. Braunschweig $\cdot$ G. O. Hofmann
}

Received: 12 October 2008/Accepted: 23 February 2009/Published online: 14 March 2009

(C) Springer-Verlag 2009

\begin{abstract}
Septic diseases of the bone and the immediate surrounding soft tissue, i.e., osteitis, belong to the most alarming findings in recent traumatology and orthopedic surgery. The paramount goal of this therapy is to preserve the stable weight-bearing bones while maintaining a correct axis and proper working muscles and joints, in order to avoid permanent disability in the patient. "State-of-the-art" therapy of osteitis/osteomyelitis therapy has two priorities: eradication of the infection and reconstruction of bone and soft tissue. Surgical treatment of the affected bone segments and soft tissue, followed by reconstructive methods,
\end{abstract}

A. H. Tiemann $(\bowtie)$

Funktionsbereich Septische und Rekonstruktive Chirurgie, Klinik für Unfall- und Wiederherstellungschirurgie,

BG-Kliniken Bergmannstrost Halle (Saale),

Merseburger Str. 165, 06112 Halle, Germany

e-mail: andreas.tiemann@bergmannstrost.com;

susann.tiemann@t-online.de

H. G. K. Schmidt

Chefarzt der Abteilung für Septische Knochen-

und Weichteilchirurgie, Klinikum Eilbek, Schön Kliniken,

Dehnhaide 120, 22081 Hamburg, Germany

R. Braunschweig

Direktor der Klinik für bildgebende Diagnostik

und Interventionsradiologie, BG-Kliniken Bergmannstrost Halle

(Saale), Merseburger Str. 165, 06112 Halle, Germany

G. O. Hofmann

Direktor der Kliniken für Unfall-, Hand-

und Wiederherstellungschirurgie, Friedrich-Schiller

Universität Jena, Erlanger Allee 101, 07747 Jena, Germany

G. O. Hofmann

Direktor der Klinik für Unfall- und Wiederherstellungschirurgie, BG-Kliniken Bergmannstrost Halle (Saale),

Merseburger Str. 165, 06112 Halle, Germany continues to be the main basic therapy. It is often extremely difficult to decide whether the affected bone segment has to be resected, or whether bone continuity can be preserved. The following paper provides strategies and guidance to help guide decisions in this complex and challenging area.

Keywords Osteitis - Segment resection · Preservation of bone continuity

\section{Introduction}

Despite the tremendous evolution of orthopedic and traumatologic methods (i.e., minimal invasive fracture treatment, biological osteosyntheses, etc.), and notwithstanding the development of new and powerful antibiotics, both acute and chronic osteitis remain greatly feared complications of bone and joint surgery. The paramount goals in the treatment of the osteitis (i.e., acute and chronic osteitis) are:

- eradication of the infection,

- preservation or reconstruction of weight-bearing bones,

- preservation or reconstruction of properly functioning joints,

- preservation or reconstruction of the surrounding soft tissue, and

- minimization of pain.

State-of-the-art therapy for osteitis is based on two guiding principles:

- radical surgical resection of any affected tissue (bone and soft tissue) combined with extensive wound debridement. Even today, surgery remains the basis of any further therapy. 
- Knowledgeably applied adjuvant therapy (i.e., local and systemic antibiotics, hyperbar oxygenation, optimized diet, etc.).

Despite recent advancements, it is difficult in some cases to estimate the size of bone segment that has to be resected in order to eradicate the infection. In other words, it is difficult to differentiate between:

- vital-vital affected-devitalized cortical bone

- vital-vital affected-devitalized cancellous bone.

There is no clear method of deciding the course of treatment-preservation of the bone continuity or the need for segment resection-unless the above distinction is properly made. This distinction allows for the appropriate choice of appropriate treatment methodologies.

Schmidt et al. (2003) showed that only the resection of $100 \%$ of the infected bone may lead to successful osteitis treatment, and that by leaving small infected or devitalized bone segments, the infection could in fact persist or may even be rekindled [1]. On the other hand, the authors pointed out that vital affected bone may recover when it is surrounded by vital, healthy soft tissue.

In order to arrive at the appropriate distinction between devitalized cortical bone and devitalized cancellous bone, there are two different diagnostic pathways.

\section{Pre-operative diagnostic investigation}

To diagnose the affected area before operating, radiological and nuclear medicine methods are extremely helpful. On the one hand, they lead to the preoperative detection of the local size of the osteitis focus. Additionally, one may also find out about secondary foci that may be located away from the intrinsic focus and, without using these methods, may stay undetected and thus untreated. The use of the single diagnostic procedures and their ranking may be handled differently and depends on the individual capabilities and skills of each radiology unit. It is undisputable that only a very close cooperation between radiologist and surgeon, the precise posing of the questions and the interdisciplinary discussion of any radiological but also clinical finding, may lead to a successful preoperative strategy (Figs. 1, 2).

\section{Intra-operative diagnostic investigation}

These diagnostic features will be used in a point-of-caretesting (POCT) and include evaluation of [1]:

- bone color

- "bone sound"

- bone texture

- quality of the cancellous bone

- surrounding soft tissue

Details about each of these areas to be evaluated are found below.

Bone color

Vital bone never has a uniform ivory color. Instead, there is always a texture, like the wavy grain in wood. At the localization of an osteotomy, minor punctate bleeding from the Haversian canals will be detectable (Fig. 3).

\section{Bone sound}

Hitting vital bone with a solid surgical instrument (e.g., an osteotom) will lead to a thudding, deadened, 'tubby' sound
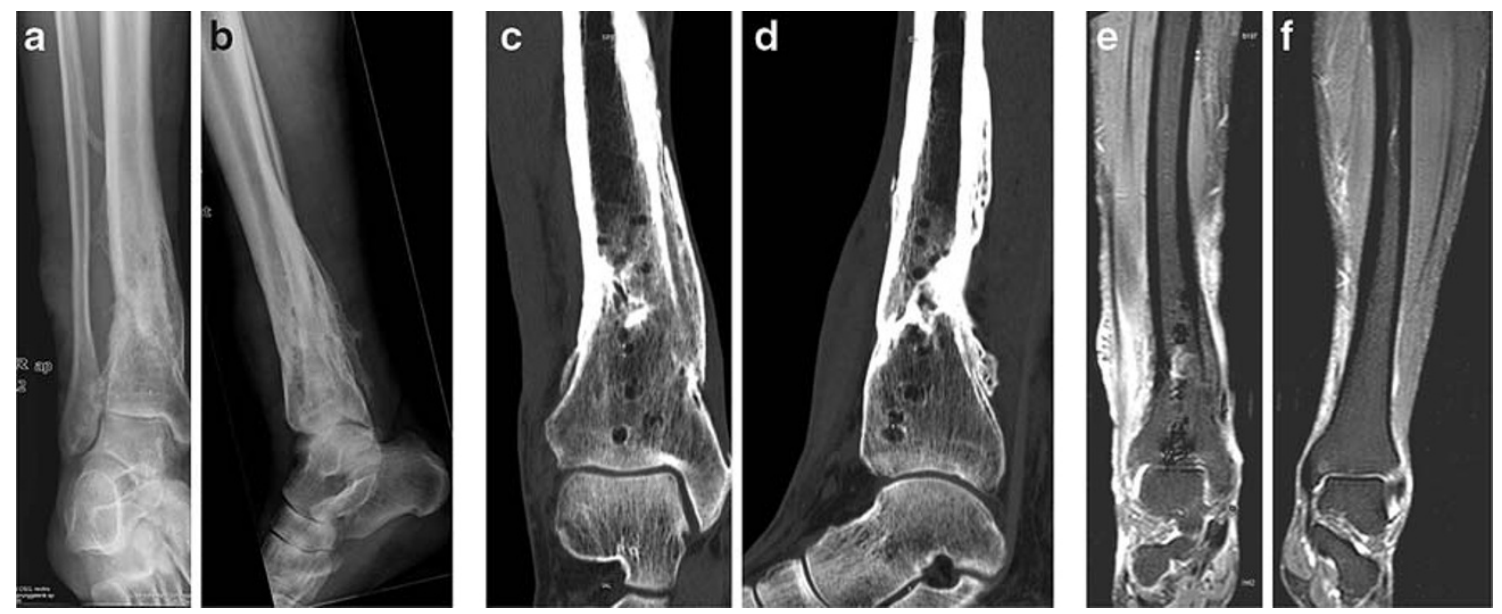

Fig. 1 Preoperative diagnostic imaging. a, b X-ray of the distal tibia: sclerosis. No signs of osteitis. c, d CT-scan of the distal tibia: sclerosis. No evidence of sequestra. $\mathbf{e}, \mathbf{f}$ MRI. No signs of an acute infection 

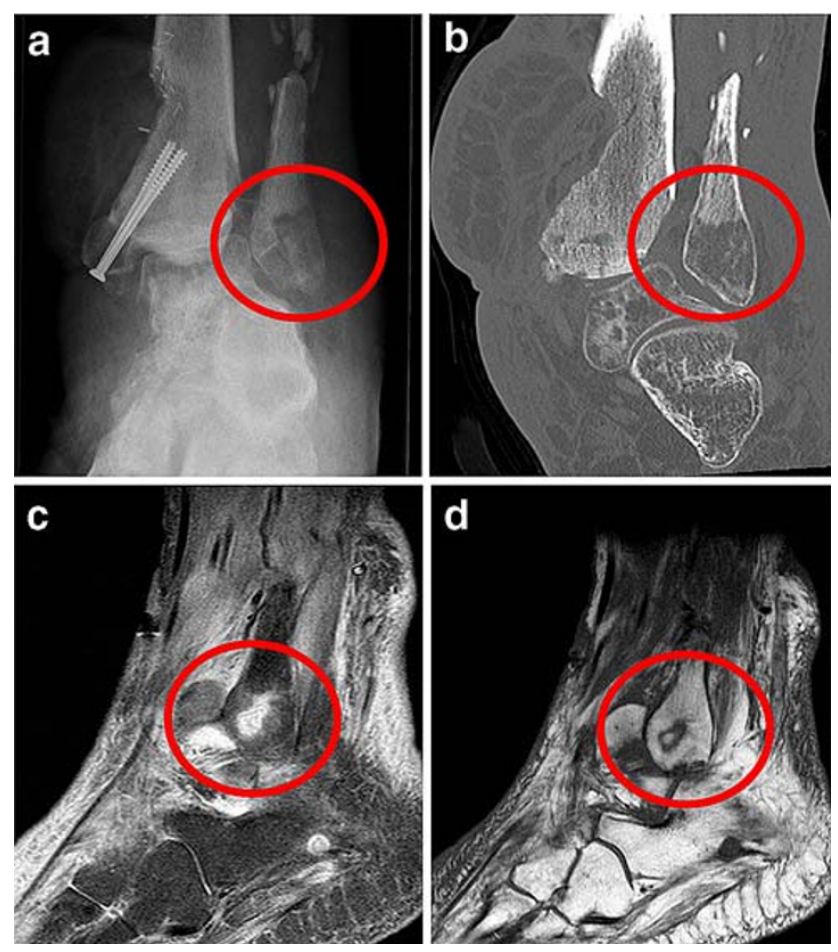

Fig. 2 Preoperative diagnostic imaging. a X-ray of the ankle: osteolytic defect of the lateral malleolus after screw fixation of a medial malleolar fracture. Destruction of the tibiotalar and the distal tibiofibular joint. b CT-scan of the ankle: defect situation distal fibula; destructed tibiotalar and tibiofibular joint. c MRI (T2-SPIR), sagittal view: edema of the distal fibula. d MRI (T1 + radiocontrast agent), sagittal view: infected area in the distal fibula

similar to the one produced by knocking on a wet log of wood. This 'bone sound' test should only be made with a solid surgical instrument in order to avoid the fact that the sound produced by the surgical instrument itself may lead to the wrong conclusion. Devitalized bone produces a clean clear sound similar the one that comes from porcelain.

Bone texture

Vital bone reacts to tangential osteotomy like living wood-it is possible to produce a chip of bone. At the localization of this osteotomy, slight bleeding from the Haversion canals will occur. Devitalized bone, on the other hand, tends to break like porcelain (Fig. 4). In order to evaluate the bone texture properly, one has to be very experienced. Particularly in the early stages of an infection, the bone may not react pathologically and this may lead to a misjudgement of the whole situation.

Quality of the cancellous bone

Again, a great deal of surgical experience is needed to tell the difference between infected and non-infected cancellous bone. Infected cancellous bone feels softer than regular cancellous bone. By taking a close look to the cancellous bone one may detect granulation tissue as well as small osseous lamellae. These tissues lead to the typical cracking sound that is produced by scraping out the bone marrow with a surgical instrument.

\section{Surrounding soft tissue}

Vital bone normally is surrounded by vital, non-infected soft tissue. Even vital affected bone may recover when the surrounding soft tissue does not show any pathological changes especially in terms of the microcirculation. It is an alarming sign if the physiologically solid combination

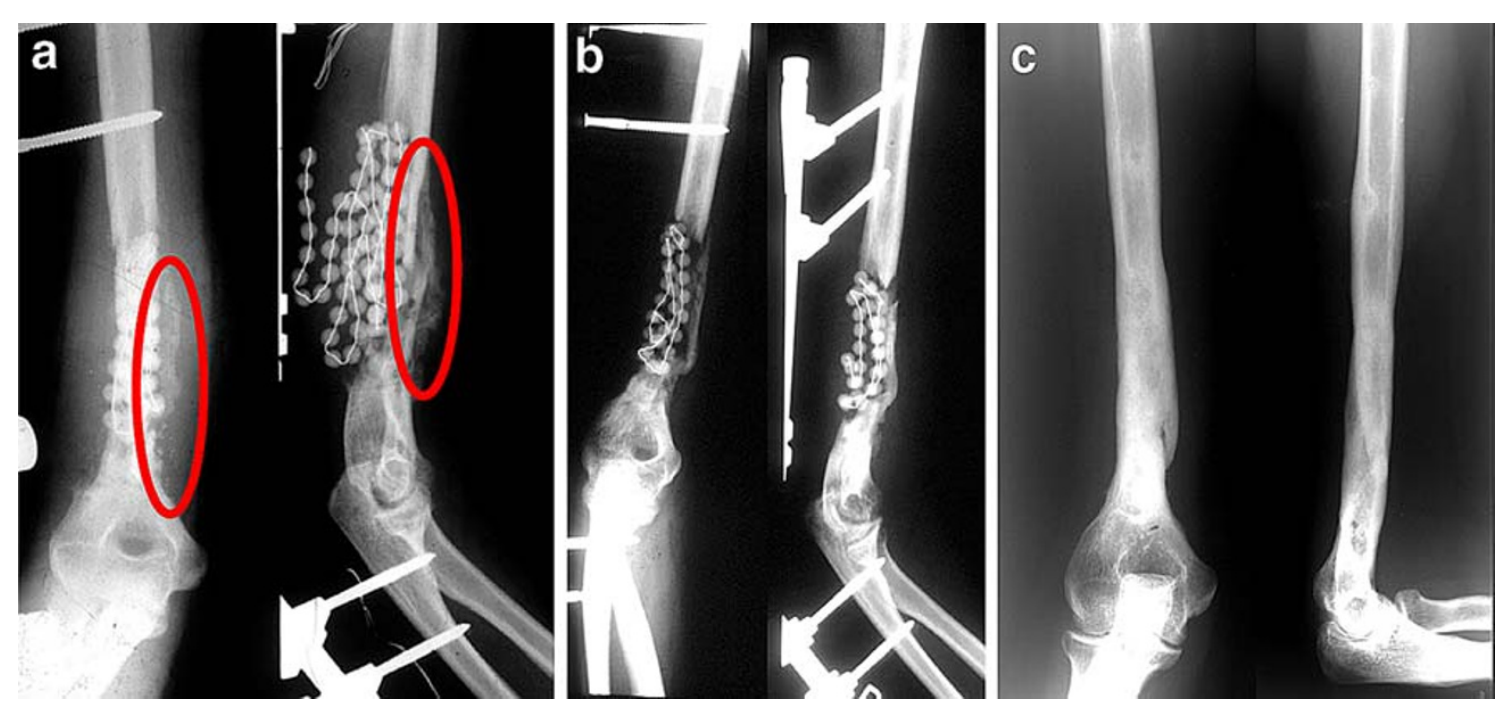

Fig. 3 Preoperative diagnostic imaging. a X-ray of the distal humerus after revision surgery due to osteitis. Persistent sequester anterolateral. b X-ray after removal of the sequester. $\mathbf{c} X$-ray after bone reconstruction with a cancellous bone graft 
Fig. 4 Vital bone. Bleeding from the Haversian canals after elevation of a "bone-chip"
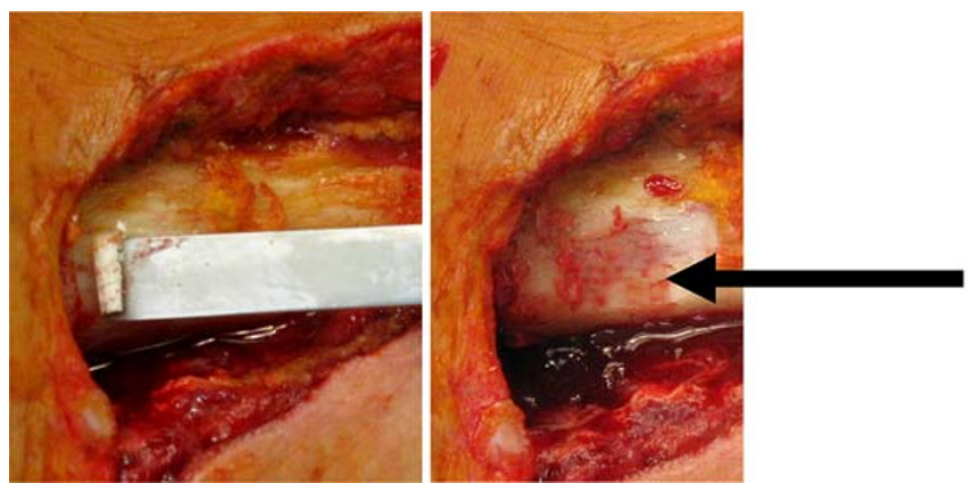

Bleeding from the Haversian canals between bone and surrounding soft tissue does not exist because in these areas the bone may be necrotic (typical locations are the dorsal aspect of the femur or the medial surface of the tibia) [1].

\section{Bone reconstructive procedures}

The use of a specific bone reconstructive procedure depends on the following three deciding factors:

- localization of the bone lesion,

- size of the bone lesions, and

- the quality of the surrounding soft tissue.

\section{Localization of the bone defect}

In 2006, Schieker et al. developed a classification system for bone defects. This classification may also be used in defects caused by osteitis [2]. The authors defined the following localizations:

- vertebral bone defect

- metaphysal bone defect

- semi-diaphyseal bone defect

- full diaphyseal bone defect.

The reconstructive options for semi- and full diaphyseal bone defects will be discussed later in this paper.

Size of the bone defect

Bone lesion size is a factor that is closely intertwined with the question of how to reconstruct the bone integrity and will be discussed below.

Quality of the surrounding soft tissue

It is of course clear that, before bone reconstructive surgery is begun, the surrounding soft tissue should be found not to contain any pathological findings (i.e., no infection, optimal micro-vascularization, etc.). In other words, the surrounding soft tissue should be in the best possible condition, a key factor in the successful bone reconstruction. If any malfunction or pathological finding is determined, appropriate procedures should be undertaken in response (i.e., surgical and antibiotic eradication of any local infection, optimizing the micro- and macro-vascularization by vascular surgical operations, etc.).

\section{Type of bone reconstructive procedures}

That autologous bone graft play a leading role after eradication and sedation of the osteitis is a readily understood fact. The use of heterologous materials may lead to the reactivation of the osteitis. Thus, bone reconstruction is based on two procedures:

- autologous cancellous bone graft and

- segment-transfer/callus distraction/callotaxis.

The autologous cancellous bone graft may be combined with growth factors (BMP), and/or antibiotic chips. As previously mentioned, the specific treatment method depends on the size of the bone defect.

\section{Semi-diaphyseal bone defect-small non-segmental diaphyseal defects}

Bone reconstruction via autologous cancellous bone graft is recommended. As discussed by Schweiberer et al. [3], the overriding clinical goal when using the cancellous bone graft next to the replenishment of the bone loss is to induce osteogenesis and to create some biomechanical stability. The cancellous bone graft may be use in the above-named combination with growth factors and/or antibiotic chips. The benefit of the defect management by antibioticimpregnated autogenic cancellous bone grafts or cancellous bone grafts combined with growth factors has been mentioned in previous papers [4-6]. 


\section{Full diaphyseal bone defect}

The method used is determined by the size of the bone defect. Nevertheless, a review of the literature shows that the size which delineates the borderline between cancellous bone plastic and callus distraction is not agreed upon. Different authors have pinpointed different sizes. For example, Schmidt et al. and Schieker et al. both alluded to $3 \mathrm{~cm}$ as the critical size, while Steinau et al. judged $4 \mathrm{~cm}$ to be the ultimate bone defect size, which one may bridge by cancellous bone grafts [1, 2, 7]. In 2000, Masquelet [8] even reported on a series of 35 cases of large diaphyseal bone defects reconstructed by autologous bone grafting. The size of the bone defects varied between 4 and $25 \mathrm{~cm}$.

\section{Influence of the "bone bed"}

In addition to the size of the bone defect, the decision to use a specific reconstructive method ultimately depends on the surrounding soft tissue. Thus, any bone reconstructive strategy depends on the biological capacity of the bone bed in terms of osteoneogenesis, osteoinduction, osteoconduction and microcirculation. In other words, the quality of the bone bed depends on it's capability to support the survival of transferred bone material and the formation of new bone. According to this, the bone bed formed by the surrounding soft tissue may be classified into three groups [2]:

- Excellent bone bed (EBB): Optimal precondition for the bone reconstruction

- Mediocre bone bed (MBB): Significantly worse precondition for the bone reconstruction

- Poor bone bed (PBB): Poor precondition for the bone reconstruction.

In cases with optimal and 'super vital' surrounding soft tissue, the borderline for the use of the callotaxis may be shifted toward $5 \mathrm{~cm}$. On the other hand, the manner of performance of the cancellous bone plastic is vitally important. Depending on the bone defect's size and the surrounding soft tissue, it may be necessary to integrate the plastic in multiple steps in order to avoid cancellous bone graft necrosis, which may reactivate the osteitis (Figs. 5, 6, 7). According to the bone bed the average diameter may vary:

- Femur + EBB: $2.5 \mathrm{~cm}$

- Femur + MBB: $1.5 \mathrm{~cm}$

- Femur + PBB: $1.0 \mathrm{~cm}$

- Tibia + EBB: $2.0 \mathrm{~cm}$

- Tibia + MBB: $1.0-1.5 \mathrm{~cm}$

- Tibia + PBB: $0.5 \mathrm{~cm}$

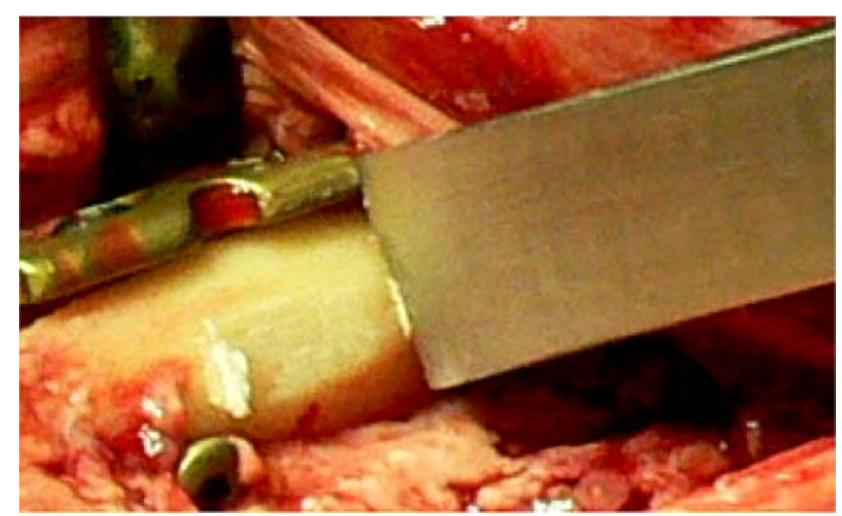

Fig. 5 Devitalized bone. No bleeding of the Haversian canals after elevation of a "bone-chip". Texture and color similar to ivory
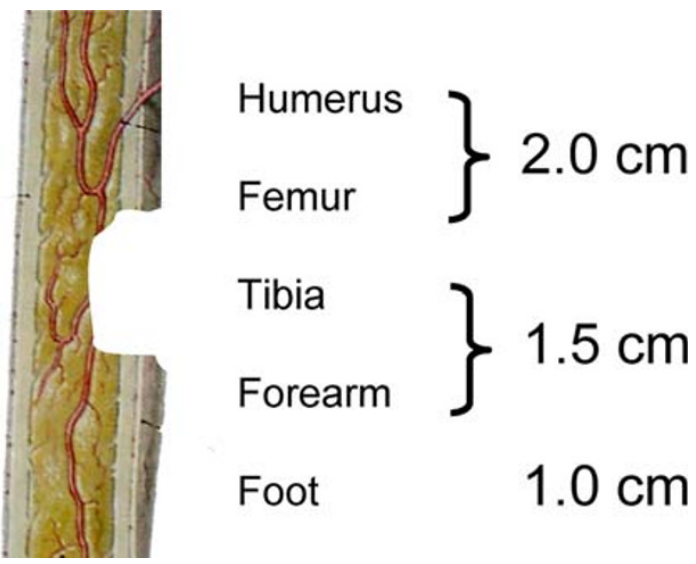

Foot

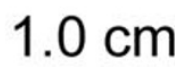

Fig. 6 Considered average diameter of cancellous bone plastic implanted in one session in order to avoid necrosis of the bone transplant $[1,12]$

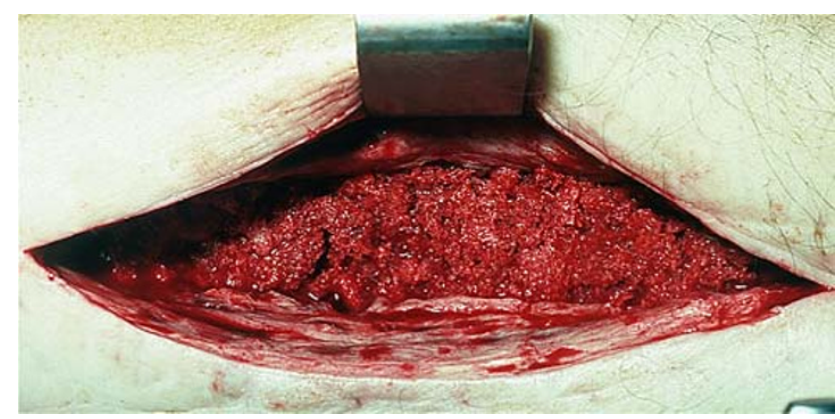

Fig. 7 Typical structure of a cancellous bone plastic

In order to mimic the natural form of bones (tube) the cancellous bone has to be integrated in layers at the outside of the bone bed from one end of the defect to the other surrounding a central coiled antibiotic fleece.

It is known that the integration of the cancellous bone graft depends on the time that is used in order to create a 
Table 1 The University of Texas Medical Branch (UTMB) staging system for adult osteomyelitis [11]

\begin{tabular}{ll}
\hline Anatomic type & Medullary osteomyelitis \\
I & Superficial osteomyelitis \\
II & Localized osteomyelitis \\
III & Diffuse osteomyelitis \\
IV & \\
Physiologic class & Good immune system and delivery \\
A-host & Compromised locally or systemically \\
B-host & Requires suppressive or no \\
C-host & treatment; minimal disability; \\
& treatment worse than disease; \\
& not a surgical candidate \\
Clinical stage & \\
Type + class $=$ clinical stage & \\
\hline
\end{tabular}

proper micro-vascularization in the transferred bone material [9]. In experimental studies, the acceleration of this microvascular in-growth was measured between 0.22 and $0.42 \mathrm{~mm} /$ day [10]. On the other hand, one may not destroy the physiological texture of the cancellous bone by compression during the transplantation. Compressed cancellous bone does contain more osteo-inductive potential, but the destroyed physiological texture avoids the proper micro-vascularization and may lead to necrosis [3].

\section{Conclusion}

The choice between autologous cancellous bone graft and callotaxis in order to reconstruct the bone integrity after eradication of the osteitis depends on the following key facts:

- type of receptor, according to the classification of Cierny et al. [11], that is based on the individual local and systemic risk-factors (Table 1),

- localization of the bone defect,

- size of the bone defect,

- quality of the surrounding soft tissue.

The following principles can serve as general guidelines for decision-making:

- small partial diaphyseal defects up to semi-diaphyseal defects or full diaphyseal defects $(<3-4 \mathrm{~cm})$, with good surrounding soft tissue $\rightarrow$ autologous cancellous bone plastic (+/- growth factors, $+/-$ antibiotic chips)

- full diaphyseal bone defect $(>3-4 \mathrm{~cm})$, impractical surrounding soft tissue $\rightarrow$ callotaxis.

Naturally, these guidelines should be adjusted to fit the individual circumstances. It is also to be understood that the borderline between bone graft and callotaxis may be shifted one way or another. However, the above guidelines provide a basis for making critical treatment choices.

Acknowledgments English proofreading by Patience Montgomery. M.A. (English as a Second Language and Bilingual Education).

\section{References}

1. Bühler M, Engelhardt M, Schmidt HGK (2003) Septische postoperative Komplikationen. Springer, Wien. ISBN 3-211-83811-2, pp. $174-186$

2. Schieker M, Mutschler W (2006) Die Überbrückung von posttraumatischen Knochendefekten. Unfallchirurg 109:715-732

3. Schweiberer L, Eitel F, Betz A (1982) Spongiosatransplantation. Chirurg 53:195-200

4. Dereka XE, Markopoulou CE, Mamalis A, Pepelassi E, Vrotsos IA (2006) Time- and dose-dependent mitogenic effect of basic fibroblast growth factor combined with different bone graft materials: an in vitro study. Clin Oral Implants Res 17:554-559

5. Lindsey RW, Probe R, Miclau T (1994) The effects of antibioticimpregnated autogenic cancellous bone graft on bone healing. Clin Orthop 291:303-312

6. Yi-Sheng C, Wen-Neng Ueng S, Wang CJ, Lee SS, Chao EK, Shin CH (1998) Management of small infected tibial defects with antibiotic-impregnated autogenic cancellous bone grafting. J Trauma 45(4):758-764

7. Schnettler R, Steinau HU (2004) Septische Knochenchirurgie Georg Thieme Verlag KG. ISBN 3-13-116981-8, pp. 117-127

8. Masquelet AC, Fitoussi F, Begue T, Muller GP (2000) Reconstruction of the long bones by the induced membrane and spongy autograft. Ann Chir Plast Esthet Jun 45(3):346-353

9. Eitel F, Schweiberer L, Saur K, Dambe LT, Klapp F (1980) Theoretische Grundlagen der Knochentransplantation: Osteogenese und Revascularisation als Leistung des Wirtslagers. In: Hierholzer G, Zilch H (eds) Transplantatlager und Implantatlager bei verschiedenen Operationsverfahren. 16. Jahrestagung der Deutschen Gesellschaft für Plastische und Wiederherstellungschirurgie in Düsseldorf. Hrsg. Springer, Berlin, S.1

10. Stringa G (1957) Studies of the vascularization of bone grafts. J Bone Joint Surg Br 39:395-420

11. Cierny G 3rd, Mader JT, Penninck JJ (1985) A clinical staging system for adult osteomyelitis. Contemp Orthop 10:17-37

12. Sobotta J, Becher H (1972) Atlas der Anatomie der Menschen. Band 1. Urban \& Schwarzenberg, München. ISBN 3-541-02817-3 